\title{
The Influence Effects of Leader Prestige Impacts on Subordinates' Job Attitude and Behavior
}

\author{
HE Li \\ (Faculty of Politics and Public Management, Guangdong University of Foreign Studies, 510006, \\ China.)
}

Keywords: leader prestige; Leader-member exchange; Leader trust; Organizational identification; Job engagement; Silence behavior

\begin{abstract}
Through the empirical research method, this study discusses leader prestige problem which the Chinese people are familiar with in many aspects. With enterprise staff as the research object, the research analyses the influence effect of leader prestige on subordinates' job attitude and behavior. The results show that leader prestige of administrators can significantly directly and signally enhance the subordinates' organizational identification and job engagement level, and inhibit the silence behavior; Leader prestige, by leader-member exchange, directly improves subordinates' organizational identification and job engagement, and indirectly generates inhibiting effect on subordinates silence behavior; Leader prestige can also use the intermediary role of trust to leaders from subordinates, increase the level of job engagement of subordinates, and reduce silence behaviors.
\end{abstract}

\section{Introduction}

Under the Chinese traditional culture, the prestige has been the important standard to test whether the subordinates will support leader/manager, is also a sign to test leadership effectiveness. In aspects of the influence of culture on leadership theory, the leadership connotation, leadership style and practice are influenced by the culture (Cheng, B. S. et al., 2003).So, it is very necessary to carry out the theory research on leader prestige of managers under the Chinese culture.

\section{Research Theory and Hypothesis}

(1) Leader prestige is a kind of influence under Chinese culture

The word "prestige" is proposed under the background of Chinese culture, containing the concept of prestige and credibility. Prestige is the potential authority and influence of people's personality, an influence without power, as well as a sincere compliment, respect and trust to the person in a certain position (Zheng Q., 2008).

At home, Qian S.H., and Min W.G., (2002) think that setting up leader prestige must strengthen the flexible management means, in addition to power and post, prestige of managers also include character, quality, knowledge, ability and other factors of managers. Zheng Q. (2008)thinks that there is constraint and non- constraint influence, the former referring to power, the latter to prestige. But one who has power does not necessarily has prestige. The source of prestige includes morality, knowledge, talent, integrity, honesty, etc. This study argues that leader prestige under Chinese culture is the non-power influence generated by the leader's own factors and behaviors, which can win endorsements and supports of subordinates.

(2)Research hypothesis

1. Effects on employees' attitudes and behaviors of leader prestige

Epitropaki et al. (2005) have shown that transformational leadership will produce positive effects on employee's organizational identity, this is because identity of the group members to the organization is to meet the identity of the organizational goal and the significance, and the leader is the communicator of organization meaning. Reade (2001) also found that the superior support has high correlation with organizational identity. At home, Zheng Q.(2008), Qian S.H. and Min W.G.(2002) and others in some relevant discourse, have analyzed the influence of leader prestige on 
the psychological and behavioral aspects of subordinate. So this research proposes the following hypothesis:

Hypothesis 1: Leader prestige has significant positive influence on organizational identification of subordinates.

Hypothesis 2: Leader prestige has significant positive influence on the subordinates' job engagement.

In addition, in all kinds of influenced sources to the silence behavior of the subordinates, leading factors are the important factors that affect employee to select recommendations or keep silent. In the face of the leaders with higher authority, subordinates should dare to break the silence and be positive to provide suggestions. Therefore, this research put forward the following hypothesis:

Hypothesis 3: leader prestige has significant negative predictive power to the subordinates' silence behavior.

2. Mediating effects of leader-member exchange

According to the theory of leader-member exchange (LMX), Graen et al. (1995) suggested that leaders would treat subordinates distinctively according to their own standard, and then form LMX with different degree of intimacy. Study of Liden \& Maslyn (1998) also found that leader-memberhip with high quality is of great influence on the subordinates' work behavior and attitude, and would lead to identity from subordinates to the organization. Therefore, this research proposes the following hypothesis:

Hypothesis 4: LMX plays mediating effects between leader prestige and organizational identification.

Hypothesis 5: LMX plays mediating effects between leader prestige and job engagement.

Hypothesis 6: LMX plays mediating effects between leader prestige and silence behavior.

3. Mediating effects of leader trust from subordinates

The study of paternalistic leadership made by Farh, J. L., Cheng, B. S. (2000) showed that kindness and virtue leadership can significantly enhance the level of the trust of subordinates to the superior, while authoritarian leadership will significantly reduce the trust level. Dirks and Ferrin (2002), according to the meta-analysis method, put forward a set of theoretical framework of "leader behavior-leader trust-employee reaction"; If subordinates trust the superior, they will tend to set up the caring relationship between each other, thus make positive working behaviors. Therefore this research proposes the following hypothesis:

Hypothesis 7: Leader trust of subordinates plays mediating effects between leader prestige and organizational identification.

Hypothesis 8: Leader trust of subordinates plays mediating effects between leader prestige and job engagement.

Hypothesis 9: Leader trust of subordinates plays mediating effects between leader prestige and subordinates'silence behavior.

\section{Research Method and Research Process}

(1) Investigation process

Multiple different types of enterprises and their regular employees are selected as respondents, and it is required that employees should work in enterprises more than one year and only have one immediate superior. The investigation gave out 600 questionnaires, recycling 408 effective questionnaires, and the recovery rate of effective questionnaire was $68 \%$.

(2) Respondents

The enterprise where respondents are covers the general manufacturing, high and new technology enterprise, the service industry and other industries. In terms of rank, the average position is of 216 people; Basic management positions have 127 people; Middle management positions have 58 people; The top management positions have 7 people.

(3) Research tool

Leader prestige questionnaire developed by "leader prestige" factor in the organizational climate scale organized by Pan X.F. and Qin Q.W. (2007), including 5 items. The scale in the study of Pan 
X.F. and others for many times has high reliability and validity. The questionnaire is evaluated by subordinates to the immediate superior. Leader-member exchange questionnaire uses LMX-7 questionnaire, including 7 items. Leader trust questionnaire uses trust questionnaire of subordinates to the leaders compiled by Podsakoff et al. (1990), including 4 items. Organizational identification questionnaire uses organizational identification questionnaire developed by Mael \& Ashforth (1992), and the questionnaire has only one dimension, including 6 items. Job engagement questionnaire uses items of Michigan organization evaluation questionnaire (MOAQ) compiled by Cammann et al. (1983), including 3 items. Silence behavior questionnaire is developed by silence behavior scale which Tangirrala and Ramanujam used (2008), including 5 items.

All the above questionnaires in the investigation used Likert seven point scoring and required the respondent to determine the compliance degree of these assessments with the actual conditions according to the description of items. 1 signifies non-compliance, and 7 means good compliance.

\section{Research Results}

(1) Confirmatory factor analysis results

First of all, six questionnaires adopted mature questionnaires, and carried on the revision in the pilot test, thus ensuring good content validity. Secondly, in order to determine different construct between the research variables with each other, confirmatory factor analysis was carried out on the above six variables. From the perspective of the confirmatory analysis results of table 1, fit index of the basic model (six factors model) is better, which shows that each questionnaire represents relatively independent construct, and has good structure validity.

Table The result of confirmatory factor analysis of concept discrimination ( $\mathrm{N}=408)$

\begin{tabular}{c|c|c|c|c|c|c|c}
\hline Model & $\chi^{2}$ & $\mathrm{df}$ & $\chi^{2} / \mathrm{df}$ & RMSEA & CFI & TLI & PNFI \\
\hline Basic model & 968.489 & 390 & 2.483 & .060 & .931 & .923 & .798 \\
\hline Model 1 & 1308.182 & 395 & 3.312 & .075 & .891 & .879 & .773 \\
\hline Model 2 & 1549.707 & 399 & 3.884 & .084 & .862 & .850 & .755 \\
\hline Model 3 & 2465.846 & 402 & 6.134 & .112 & .753 & .732 & .665 \\
\hline Model 4 & 3458.549 & 404 & 8.561 & .136 & .634 & .606 & .563 \\
\hline Model 5 & 4017.944 & 405 & 9.921 & .148 & .567 & .535 & .505 \\
\hline
\end{tabular}

Note: PRE represents leader prestige, LMX represents LMX, TRU represents leader trust, ORG represents organizational identification, INV represents job engagement, and SIL represents silence behavior;+ represents factors combined.

Basic model: 6 factors: PRE, LMX, TRU, ORG, INV, SIL

Model 1: 5 factors: PRE, LMX+TRU, ORG, INV, SIL

Model 2: 4 factors: PRE, LMX+TRU, ORG+INV, SIL

Model 3: 3 factors: PRE, LMX+TRU, ORG+INV+SIL

Model 4: 2 factors: PRE+LMX+TRU, ORG+INV+SIL

Model 5: 1 factor: PRE+LMX+TRU+ORG+INV+SIL

(2) Descriptive analysis on each variable

The result in Table 2 shows the correlation of all the variables comply with the research expectation. Leader prestige has the significant correlation between the mediating variables such as LMX, leader trust. Meanwhile mediating variables and consequence variables also are related significantly. In addition, Cronbach $\alpha$ of all the questionnaire is above 0.75 , which has the high reliability.

Table 2 Correlation of descriptive analysis results $(\mathrm{N}=408)$

\begin{tabular}{l|c|c|c|c|c|c|c|c}
\hline & $\mathrm{M}$ & $\mathrm{SD}$ & 1 & 2 & 3 & 4 & 5 & 6 \\
\hline 1.Leader prestige & 5.583 & 1.163 & $(.940)$ & & & & & \\
\hline 2. Exchange relation & 5.083 & 1.109 & $.582^{* *}$ & $(.920)$ & & & & \\
\hline 3. Leader trust & 5.479 & 1.236 & $.652^{* *}$ & $.747^{* *}$ & $(.909)$ & & & \\
\hline 4. Organization identification & 5.316 & .986 & $.388^{* *}$ & $.482^{* *}$ & $.414^{* *}$ &.$(.799)$ & & \\
\hline 5. Job engagement & 5.352 & 1.118 & $.333^{* *}$ & $.463^{* *}$ & $.421^{* *}$ & $.473^{* *}$ & $(.774)$ & \\
\hline 6. Silence behavior & 3.242 & 1.222 & $-.206^{* *}$ & $-.227^{* *}$ & $-.205^{* *}$ & $-.142^{* *}$ &.$-.262^{* *}$ & $(.877)$ \\
\hline
\end{tabular}


Note: ** is $\mathrm{P}<0.01$, ${ }^{*}$ is $\mathrm{P}<0.05$, the same below; Numbers on the diagonal braces are Cronbach a of the scale

(3) Direct effects on consequence variables of leader prestige

At the beginning of the study, the latent variable path analysis method is used to verify the direct relationship between leader prestige and organizational identification, job engagement and silence behavior of subordinates. Fit index reached a better level $\left(\chi^{2}=508.910, \mathrm{df}=149, \chi^{2} / \mathrm{df}=3.416, \mathrm{CFI}=0.920, \mathrm{TLI}=0.908, \mathrm{PNFI}=0.776, \mathrm{RMSEA}=0.077\right) . \quad$ Results of path analysis showed that leader prestige have significant positive influence on organizational identification and job engagement of subordinates, the path coefficients were $0.44(\mathrm{P}<0.001), 0.35$ $(\mathrm{P}<0.001)$, and it has significant negative impact on silence behavior, the path coefficient was 0.24 $(\mathrm{P}<0.001)$.Therefore, hypothesis 1, 2, 3, got the empirical support.

(4) Inspection of leader prestige mechanism

Using the latent variable path analysis method compares with a set of nested model of hypothesis, and chooses relatively frugal model with better data fitting. According to the hypothesis model $\Delta \chi^{2}$ (degree of freedom is $\Delta \mathrm{df}$ ) to judge the advantages and disadvantages.

Model 1 is the original model of the assumption, the mediating relationship model between LMX and leader trust. In model 1, the path from LMX to silence behavior, and the path of leader trust to organizational identification are not significant, therefore they are eliminated, getting model 2; Model 3, on the basis of hypothesis model, at the same time increases the direct path from leader prestige to organizational identification, job engagement, silence behavior; Model 4 to 6 , on the basis of hypothesis model, respectively and separately increases the direct path from leader prestige to organizational identification, job engagement and silence behavior. $\Delta \chi^{2}(\Delta \mathrm{df})$ of the model 2 and 1 are compared with; Model 3-6 $\Delta \chi^{2}(\Delta \mathrm{df})$ value was compared with that of model 2, by the table, the degree of fit of the model to the data has not been reached significant level $(\Delta \chi 2)$, shown in table 3 . According to the fit index and frugality principle, model 2 is the optimal model, as shown in figure 1 .

Table 3 Fit index of each model $(\mathrm{N}=408)$

\begin{tabular}{c|c|c|c|c|c|c|c|c}
\hline Model & $\chi^{2}$ & $\mathrm{df}$ & $\chi^{2} / \mathrm{df}$ & RMSEA & CFI & TLI & PNFI & $\Delta \chi^{2}(\Delta \mathrm{df})$ \\
\hline Model 1 & 1130.170 & 395 & 2.861 & .068 & .912 & .903 & .791 & - \\
\hline Model 2 & 1131.991 & 397 & 2.851 & .067 & .912 & .903 & .795 & $1.821(3)$ \\
\hline Model 3 & 1125.370 & 394 & 2.856 & .068 & .912 & .903 & .790 & $6.621(3)$ \\
\hline Model 4 & 1129.149 & 396 & 2.851 & .067 & .912 & .903 & .793 & $2.842(1)$ \\
\hline Model 5 & 1131.865 & 396 & 2.858 & .068 & .912 & .903 & .793 & $0.126(1)$ \\
\hline Model 6 & 1128.488 & 396 & 2.850 & .067 & .912 & .904 & .793 & $3.503(1)$ \\
\hline
\end{tabular}

Model 1: hypothesis model;

Model 2: remove the path of LMX to silence behavior, leader trust to organizational identification;

Model 3: on the basis of model 2, at the same time increase the direct path from leader prestige to organizational identification, job engagement and silence behavior;

Model 4: on the basis of model 2, adding the direct path from leader prestige to organizational identification;

Model 5: on the basis of model 2, adding the direct path from leader prestige to job engagement;

Model 6: on the basis of model 2, adding the direct path from leader prestige to silence behavior;

$\Delta \chi^{2}(\Delta \mathrm{df})$ in the model 2 are the comparison of the model 2 and model $1 ; \Delta \chi^{2}(\Delta \mathrm{df})$ in the model 3-6 are the comparison of the model 3-6 and model 2 . 


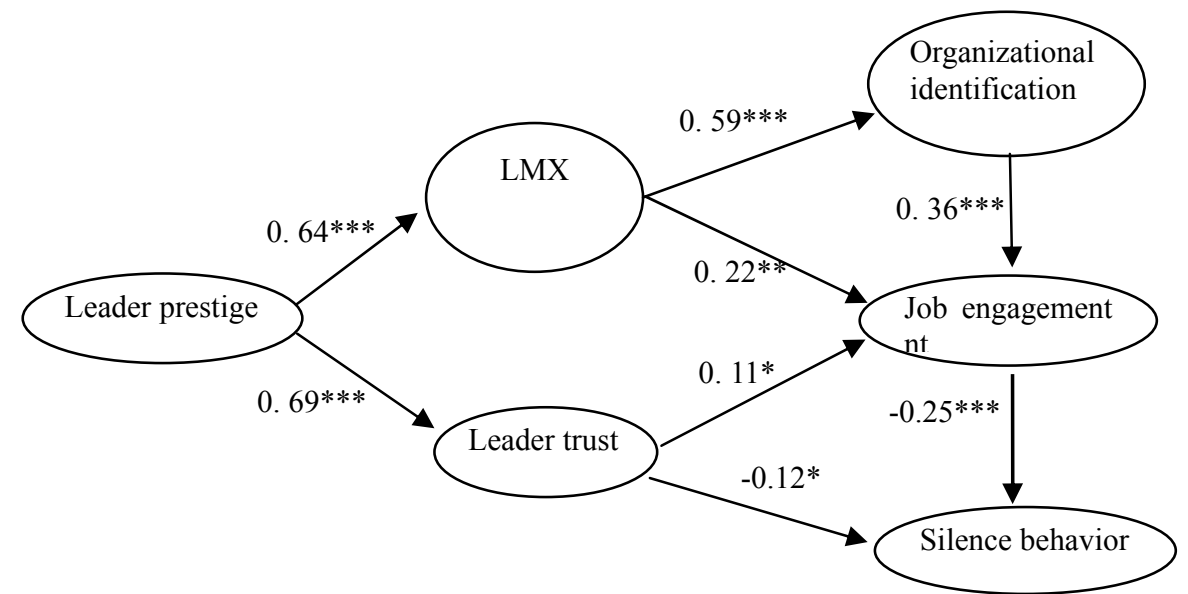

Figure 1 Correction model of leader prestige and its relevant variables (model 2)

The above result shows: (1) leader prestige has significant positive influence on LMX and leader trust, $(\beta=0.64, \mathrm{P}<0.001 ; \beta=0.69, \mathrm{P}<0.001)$; $(2)$ the $\mathrm{LMX}$ have significant impact on employees' organizational identification and job engagement $(\beta=0.59, \mathrm{P}<0.001 ; \beta=0.22, \mathrm{P}<$ $0.001)$;(3) Leader trust has significant positive influence on employees' job engagement $(\beta=0.11, P$ $<0.05)$, and has significant negative influence on silence behavior $(\beta=-0.12, \mathrm{P}<0.05)$; (4) Organizational identification has significant positive effect on their job engagement (beta $=0.36, \mathrm{P}$ $<0.001)$, and job engagement has negative influence on their silence behavior $(\beta=-0.25, \mathrm{P}<$ 0.001).Thus, LMX and leader trust have full mediating effects on the effect of leader prestige on organizational identification, job engagement and silence behavior of employees. So the hypothesis $4,5,6,8,9$ were confirmed, and hypothesis 7 has not been confirmed.

\section{Research Conclusion and Prospect}

First of all, this study confirmed that leader prestige has significant positive effect on the subordinates leader trust and LMX. When managers have higher leadership credibility, the trust level of subordinates to superior is higher. The effectiveness of leader prestige also need good LMX to be reflected. This suggests that leader prestige can influence the psychological and behavior of subordinates through the effective management behavior.

Second, the results proved the full mediating effects between leader trust and LMX with subordinates' organizational identification, job engagement and silence behaviors. The result shows that if leaders have higher prestige, subordinates will strengthen the sense of trust and acceptance for the leader, making subordinates believe that they can win a better reward through hard work. So they can show reactions such as higher organizational identification and positive job engagement. This process is consistent with requirements which Chinese cultural emphasis on , "insist on having both ability and political integrity, pay attention to performance, the masses accepted".

Third, the study confirmed that leader prestige has significant inhibitory effect on silence behavior of subordinates, when leader prestige of the managers is higher, subordinates will be able to break the silence and offer advice.

It is visible that in the process of the influence of leader prestige, the superior managers use their influence and personality charm to influence and lead subordinates' psychology and behavior. In management practice, managers should be having both ability and political integrity, promote character, ability and performance, set up the prestige in the subordinates, establish a good relationship between higher and lower, and can get subordinates' trust for managers and identification of the organization, thus improving job engagement of subordinates, and actively put forward advice behavior, which plays an important role in the construction of harmonious organizations with high performance. 
Project funding: MOE (Ministry of Education in China) Youth Project of Humanities and Social Sciences (Project No. 10YJC630074)," The Study on the Content Structure and Influence Mechanism of Leader Prestige"; Introducing Talent Project of Guangdong University of Foreign Studies.

Author introduction: 【He Li (1978-), male, Xinfeng county in Jiangxi province, associate professor, PHD, Research direction: Organizational behavior and human resource management 】

\section{References}

[1] Fan, J.L., Zheng, B.X. Paternalistic Leadership Organized by Chinese Organization: Analysis on A Cultural Opinion [J]. Research on Local Psychology, 2000, 13: 127 180

[2] Pan, X.F., Qin, Q.W. Measurement of Organizational Atmosphere in Middle School and Relevant Analysis on Psychological Health of Teachers [J]. Psychological Science, 2007, 30 (4): 982-986.

[3] Qian, S.H., Min, W.G. Brief Discussion on Flexible Management and Establishment of Modern Leader prestige [J]. Academic Exploration, 2002, 3.

[4] Zheng, B.X., Zhou L.F., Huang M.P., etc. Ternary model of paternalistic leadership: evidence of Chinese mainland business organization [J]. Journal of Indigenous Psychology Research, 2003, 20:209-252.

[5] Zheng, Q. Power and Prestige of Leader Cadres — Reflection on Leader Power and Prestige [J]. Journal of HuNan Industry Polytechnic, 2008, 8 (2). 